Research paper

\title{
Naturally Leishmania infantum-infected dogs display an overall impairment of chemokine and chemokine receptor expression during visceral leishmaniasis
}

\author{
Manuela S.L. Nascimento ${ }^{a}$, Talyta D.R. Albuquerque ${ }^{b}$, \\ Maria A. Do-Valle-Matta ${ }^{\mathrm{e}}$, Ivo S. Caldas ${ }^{\mathrm{f}}$, Lívia F. Diniz ${ }^{\mathrm{f}}$, André Talvani ${ }^{\mathrm{f}}$, \\ Maria T. Bahia ${ }^{\text {f }}$, Cléber M. Andradeg ${ }^{g}$, Lúcia M.C. Galvão ${ }^{\text {, }}$, Antônia C.J. Câmara ${ }^{\text {, }}$, \\ Paulo M.M. Guedes ${ }^{\mathrm{b}, *}$ \\ a Faculdade de Medicina de Ribeirão Preto, Universidade de São Paulo, Ribeirão Preto, São Paulo, Brazil \\ b Departamento de Microbiologia e Parasitologia, Universidade Federal do Rio Grande do Norte, Natal, Rio Grande do Norte, Brazil \\ c Programas de Pós-Graduação em Ciências Farmacêuticas e Ciências da Saúde, Universidade Federal do Rio Grande do Norte, Natal, Rio \\ Grande do Norte, Brazil \\ d Departamento de Análises Clínicas e Toxicológicas, Universidade Federal do Rio Grande do Norte, Natal, Rio Grande do Norte, Brazil \\ e Laboratório de Ultra-estrutura Celular, Instituto Oswaldo Cruz/FIOCRUZ, Rio de Janeiro, Brazil \\ f Departamento de Ciências Biológicas/NUPEB, Universidade Federal de Ouro Preto, Ouro Preto, Minas Gerais, Brazil \\ g Faculdade de Ciências da Saúde, Universidade do Estado do Rio Grande do Norte, Mossoró, Rio Grande do Norte, Brazil
}

\section{A R T I C L E I N F O}

\section{Article history:}

Received 10 December 2012

Received in revised form 15 February 2013

Accepted 26 February 2013

\section{Keywords:}

Canine visceral leishmaniasis

Chemokines

Chemokine receptors

Pathogenesis

Clinical manifestations

Dog

\begin{abstract}
A B S T R A C T
Dogs are the primary reservoir for Leishmania parasites. The immune response induced by Leishmania infantum infection in these animals has not been completely elucidated, and few studies have investigated the relationship between the expression levels of chemokines and chemokine receptors and the clinical status of dogs with canine visceral leishmaniasis (CVL). The aim of this study was to correlate the clinical status of naturally L. infantuminfected dogs (from rural areas of Mossoró city, State of Rio Grande do Norte, Brazil) with the expression levels of chemokines (ccl1, ccl2, ccl3, ccl4, ccl5, ccl17, ccl20, ccl24, ccl26, cxcl9, cxcl10) and chemokine receptors (cxcr3, ccr3, ccr4, ccr5, ccr6, ccr8) in the liver and spleen determined using real-time PCR. Twenty-one dogs were clinically evaluated and classified as asymptomatic $(n=11)$ or symptomatic $(n=10)$. Splenomegaly, weight loss and onychogryphosis were the most pronounced symptoms. In the liver, the mRNA expression levels of ccl1, ccl17, ccl26, ccr3, ccr4, ccr5, ccr6, and ccr8 were lower in symptomatic animals than in asymptomatic animals. Compared with uninfected animals, symptomatic dogs had lower expression levels of almost all molecules analyzed. Moreover, high clinical scores were negatively correlated with ccr5 and ccr6 expression and positively correlated with cxcl10 expression. We conclude that the impairment of the expression of chemokines and chemokine receptors results in deficient leukocyte migration and hampers the immune response, leading to the development of disease.
\end{abstract}

(C) 2013 Elsevier B.V. All rights reserved.

\footnotetext{
* Corresponding author at: Department of Microbiology and Parasitology, Federal University of Rio Grande do Norte, Av Salgado Filho SN, Lagoa Nova, 59072-970 Natal, RN, Brazil. Tel.: +55 8432119210.

E-mail address: pauloguedes@cb.ufrn.br (P.M.M. Guedes).
}

\section{Introduction}

Visceral leishmaniasis $(\mathrm{VL})$ is a neglected disease caused by the intracellular protozoan parasite Leishmania infantum, which is endemic in Brazil, and by Leishmania donovan (Mauricio et al., 2000). These parasites are transmitted via 
the bite of a female sand fly (Ashford, 2000). This infectious disease represents an important public health problem around the world, primarily in under-developed and developing countries, and the range of the causative parasite is expanding due to the rural exodus, the increased population density, the adaptation of the vector to the urban environment, and the poor conditions in urban peripheries (Mott et al., 1990; Nascimento et al., 2008).

Domestic dogs are the major reservoir of the parasites responsible for human infections (Desjeux, 2004; Moreno and Alvar, 2002) because these animals share a habitat with humans and have a high prevalence of VL. In addition, infected dogs often have high levels of cutaneous parasitism. For these reasons, dogs are responsible for the maintenance of the parasite in endemic foci (Giunchetti et al., 2006; Travi et al., 2001). Therefore, dogs are considered one of the strategic targets for disease control (Dietze et al., 1997). The wide spectrum of symptomatological profiles in canine visceral leishmaniasis (CVL) ranges from self-limiting infections to asymptomatic and oligosymptomatic forms to fatal disease. The evolution of the disease depends on the parasite's virulence, the host's genetic susceptibility (Reiner and Locksley, 1995) and the immunological mechanisms triggered after infection. This last factor is not currently well understood.

In a canine model, it was demonstrated that asymptomatic dogs express high levels of il-2, il-12, ifn- $\gamma$ and tnf- $\alpha$ mRNAs and have lower parasite burdens in the prescapular lymph nodes, the peripheral blood and the skin (Alves et al., 2009; Barbosa et al., 2011; Chamizo et al., 2005; Menezes-Souza et al., 2011). In contrast, susceptible dogs develop an adaptive immune response biased toward the Th2 profile, with the upregulation of il-4, il10 and tgf- $\beta$ mRNA production, which is correlated with a high parasite burden (Alves et al., 2009). Chemokines and their receptors are often differentially induced in response to various stimuli or pathogens. Previous investigations have revealed that the expression levels of the ccl2, ccl4, ccl5, and ccl21 mRNAs in the skin of naturally $L$. infantum-infected dogs are associated with the immunopathogenesis of CVL (Menezes-Souza et al., 2012). Other authors have demonstrated that the $\mathrm{cxcl} 10$ and $\mathrm{ccl} 5$ mRNAs are highly expressed in the spleens of experimentally infected beagles (Strauss-Ayali et al., 2007). However, the expression levels of chemokines and chemokine receptors in the livers of symptomatic and asymptomatic dogs and the correlations between these expression levels and the pathogenesis or protection against $L$. infantum infection have not been documented in the literature. Considering the critical role of chemokines and their receptors in inducing the migration of the appropriate cell types during inflammatory responses, this study was designed to provide additional information about the profile of chemokines and chemokine receptors in the spleens and livers of naturally $L$. infantum-infected dogs and to correlate these profiles with symptomatological status. In this study, we showed that alopecia, dry exfoliative dermatitis, lesions of the ear tip, onychogryphosis, weight loss and splenomegaly occur in at least $50 \%$ of animals; the last three are the most frequent clinical signs.
Additionally, symptomatic dogs had downregulated levels of ccl1, ccl17, ccl26, ccr8, ccr4, ccr6, ccr3, cxcr3 and ccr5 in the hepatic tissue compared with asymptomatic dogs.

\section{Materials and methods}

\subsection{Experimental animals}

Twenty-one mixed-breed adult dogs of both sexes, 2-8 years old, were selected from the Center for Zoonosis Control in Mossoró City, Rio Grande do Norte State, Brazil, an area endemic for CVL. The dogs used in this study were stray or domiciled mongrel dogs and were selected based on the results of serological testing using a positive indirect immunofluorescence assay test (IFAT/titer 1:40) and ELISA. ELISA plates were sensitized with $L$. infantum antigen prepared by alkaline extraction of parasites grown in Schneider medium supplemented with 20\% fetal bovine serum. The parasites were harvested in stationary growth phase. The sera (titer, 1:80) were added to the plates and antibody binding was detected using peroxidase-labeled anti-dog IgG $(1: 15,000)$ (Bethyl Laboratories, Montgomery, TX, USA). A positive parasitological diagnosis (by imprinting) based on spleen and liver tissues confirmed the infection. Six age-matched uninfected dogs were used as controls and obtained from the kennels of the Universidade Federal de Ouro Preto (UFOP). All procedures and experimental protocols were conducted in accordance with the procedures issued by the Brazilian College of Animal Experimentation (COBEA) and were approved by the Ethics Committee in Animal Research at UFRN and UFOP. Control animals (uninfected) were provided with commercial dog food and water ad libitum. Prior to the study, the dogs were dewormed and immunized against parvovirosis, leptospirosis, distemper, parainfluenza and hepatitis (Vanguard $^{\circledR}$ HTLP 5/CV-L vaccine; Pfizer, New York, NY, USA). Sera and tissue specimens from the spleen and liver were obtained from these dogs. These animals had negative IFAT results at 1:40 and negative parasitological test results for Leishmania.

\subsection{Macroscopic evaluation}

All animals were euthanized by an intravenous injection of thionenbutal (Abbott, São Paulo, Brasil) at $0.5 \mathrm{~mL} / \mathrm{kg}$ of body weight $(0.03 \mathrm{~g} / \mathrm{ml}$ in $0.8 \%$ saline solution) and potassium chloride. Necropsy was performed, and the liver and spleen were collected. The presence of splenomegaly, weight loss, onychogryphosis, lesions of the ear tip, dry exfoliative dermatitis, alopecia, mucosal pallor, hepatomegaly, cutaneous ulcerations, and keratoconjunctivitis was evaluated.

\subsection{Total RNA isolation, CDNA synthesis and real-time $P C R$}

Total RNA from liver and spleen tissues (30-35 mg) was isolated using $0.5 \mathrm{~mL}$ of TRIZOL reagent (Invitrogen) and the SV Total RNA Isolation System (Promega, Madison, WI, USA) according to the manufacturer's instructions. 
Table 1

The sequences of the primers were designed based on nucleotide sequences in the GenBank Database and were used as follows.

\begin{tabular}{|c|c|}
\hline Target & Sense and antisense sequences \\
\hline \multirow[t]{2}{*}{ CCL1 } & ACTTTTCAGAGAAGAGGATCGC \\
\hline & TGGAGCTGGTGTGTTTGTAACA \\
\hline \multirow[t]{2}{*}{ CCL2 } & TAAAAGAGTCACCAGCAGCAA \\
\hline & TTTAGGACGGTCTTGAAGATCA \\
\hline \multirow[t]{2}{*}{ CCL3 } & CAAGCAGATTCCACGCAAGTT \\
\hline & TAATACCGGGCTTGGAGCAT \\
\hline \multirow[t]{2}{*}{ CCL4 } & TGACCGTCCTTTCTCTCCTT \\
\hline & GATCTGAACCCATTGGTGCT \\
\hline \multirow[t]{2}{*}{ CCL5 } & AAGGGCTGACTGATAAATGTGA \\
\hline & AGCGAGAATTTTAATGGAAAGC \\
\hline \multirow[t]{2}{*}{ CCL17 } & CCATCGTGTTTGTAACTGTCCA \\
\hline & AATATCTGACCGCCTTCTTCAC \\
\hline \multirow[t]{2}{*}{ CCL20 } & TATGGTTCCTCCCGGATCTATC \\
\hline & TCATTGGCCAGCTGTTGTGT \\
\hline \multirow[t]{2}{*}{ CCL24 } & CCTGCTGCATGTTCTTCATTTC \\
\hline & TTCTGGTTCTTCTTGGTGGTGA \\
\hline \multirow[t]{2}{*}{ CCL26 } & TCTTCATCCTGAGTGTCCATCG \\
\hline & AGCAGAACTTGGCCACATCA \\
\hline \multirow[t]{2}{*}{ CXCL9 } & CAGATGGTCCTTAAGCCACTTT \\
\hline & ССТTTСССТGTGAАССТСАA \\
\hline \multirow[t]{2}{*}{ CXCL10 } & CACATGTTGAGATCATTGCCAC \\
\hline & TTCAGACATCTTTTCTCСССА \\
\hline \multirow[t]{2}{*}{ CCR3 } & CTAGCAGCCTCCCCTGAATTTA \\
\hline & TGTTCAGACTCCTTTTGGGACT \\
\hline \multirow[t]{2}{*}{ CCR4 } & TTTGGACTAGGTCTCTGCAAGA \\
\hline & AAAAGCCCACCAGGTACATC \\
\hline \multirow[t]{2}{*}{ CCR5 } & TGTGTCTGCTTCAAAAGCCC \\
\hline & TCACTTGTCACCACCCCAAA \\
\hline \multirow[t]{2}{*}{ CCR6 } & AGCTGTTTGTGCCAATTGCTTA \\
\hline & AAAATATTGCCCAGGAGGCC \\
\hline \multirow[t]{2}{*}{ CCR8 } & TGATATCATCTCAAGCCCCTG \\
\hline & AGCAACTTGCTGTCTCTTTGGA \\
\hline \multirow[t]{2}{*}{ CXCR3 } & TTCTTTGCCATCCCAGATTTC \\
\hline & ATGCATGGCATTTAGGCG \\
\hline \multirow[t]{2}{*}{$\beta$-actin } & ССАСТTТССТGTСТTАСССАA \\
\hline & AATTAACCACCCACGGTGTT \\
\hline
\end{tabular}

The RNA yield and the ratio of the absorbances at $260 \mathrm{~nm}$ and $280 \mathrm{~nm}\left(A_{260} / A_{280}\right.$ ratio) were determined using a NanoVue Plus Spectrophotometer (GE Healthcare, Little Chalfont, UK). The cDNA was synthesized using $1 \mu \mathrm{g}$ of total RNA using a reverse transcription reaction (ImPromII $^{\mathrm{TM}}$ Reverse Transcriptase, Promega, Madison, WI, USA). SYBR Green Mix-based real-time quantitative PCR assays were performed using a StepOnePlus ${ }^{\mathrm{TM}}$ Real-Time PCR System (Applied Biosystems, Foster City, CA, USA). The mean $\mathrm{Ct}$ values from triplicate measurements were used to calculate the expression level of the target gene, normalized to the expression level of the housekeeping gene $\beta$-actin, using the $2^{-\Delta \Delta \mathrm{Ct}}$ formula. The sequences of the primers used listed available in Table 1.

\subsection{Statistical analysis}

Data are expressed as the mean \pm standard error of the mean (SEM) and were analyzed using Student's $t$-test for nonparametric distributions and the Mann-Whitney test. All analyses were performed using PRISM 3.0 software 292 (GraphPad, San Diego, CA, USA), and significance was accepted at $p<0.05$.

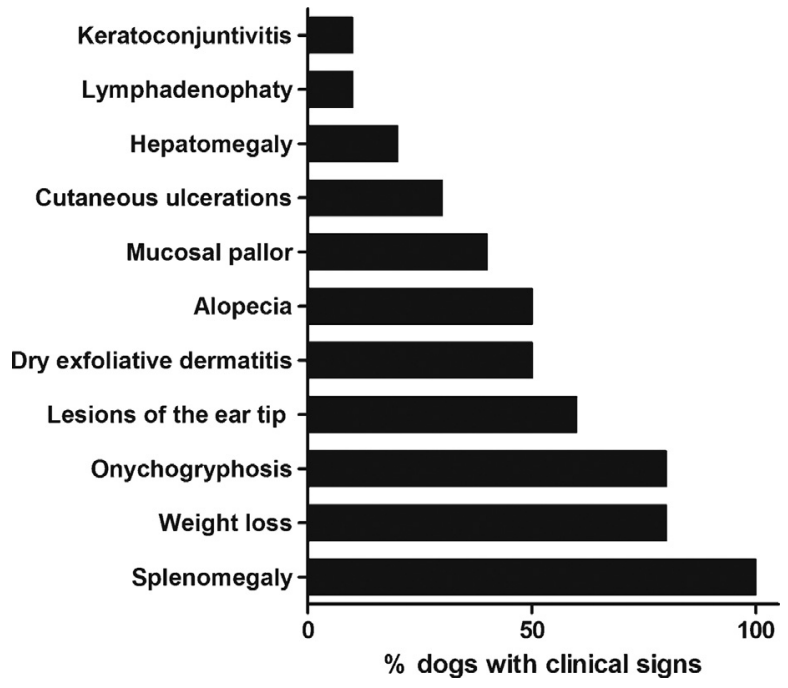

Fig. 1. Frequency of clinical signs observed in animals with CVL.

\section{Results}

3.1. Splenomegaly, weight loss and onychogryphosis are the most common symptoms of CVL

After serological confirmation of CVL in dogs, the owners were notified, and the animals collected by the Center for Zoonosis Control (CCZ) Mossoró, State of Rio Grande do Norte, Brazil. Prior to euthanasia, the animals were clinically evaluated and classified as asymptomatic (11 animals) or symptomatic (10 animals). Six uninfected dogs, obtained from the kennels of the Universidade Federal de Ouro Preto (UFOP), Ouro Preto, Minas Gerais State, Brazil, were used as controls, for a total of 27 animals. The clinical signs observed were splenomegaly, weight loss, onychogryphosis, lesions of the ear tip, dry exfoliative dermatitis, alopecia, mucosal pallor, hepatomegaly, cutaneous ulcerations and keratoconjunctivitis. The prevalences of these clinical signs in symptomatic animals are presented in Fig. 1. The only symptom observed in $100 \%$ of symptomatic animals was splenomegaly; weight loss and onychogryphosis were present in $80 \%$ of these dogs (Fig. 1).

After euthanasia, tissue samples from asymptomatic and symptomatic animals were collected for parasitological confirmation. We observed that both groups of animals had high numbers of amastigote parasites in the spleen (Fig. 2) and liver (data not shown), confirming the infection.

\subsection{Symptomatic dogs exhibit an overall downregulation of the expression of chemokines and chemokine receptors}

To understand the immunological mechanisms that determine susceptibility/resistance to L. infantum in dogs, we measured the relative quantities of the mRNAs of the chemokines and chemokine receptors related to the migration of several types of immune cells to the liver and spleen, the main target organs of L. infantum, in naturally infected dogs. In the liver tissue from symptomatic dogs, we founded a significant downregulation of the expression 

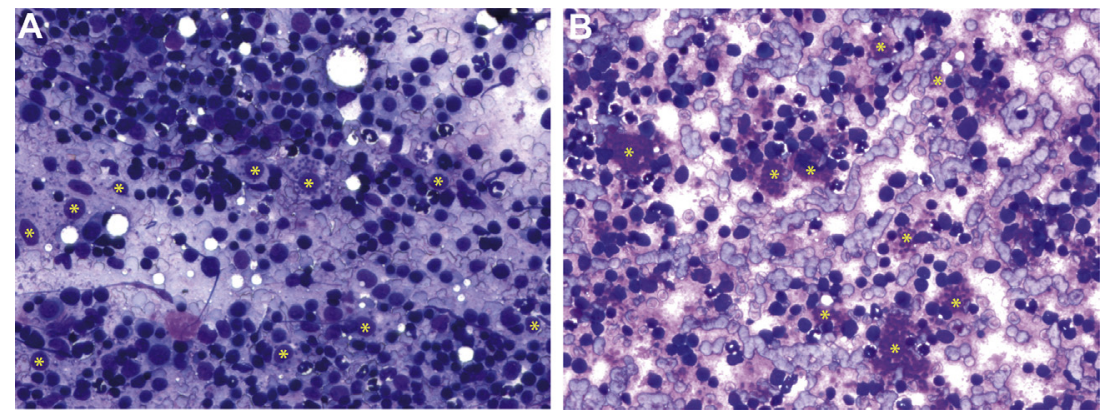

Fig. 2. Spleen imprinting for parasitological confirmation of infection. After euthanasia, samples of splenic tissue were collected for parasitological confirmation of infection. (A) Representative imprint for an asymptomatic dog. Yellow asterisks show infected macrophages. (B) Representative imprint for a symptomatic dog. Yellow asterisks show amastigote forms of the parasite. Slides show images with a $40 \times$ magnification. (For interpretation of references to color in this figure legend, the reader is referred to the web version of this article.)

of $c$ l1 $(p=0.0273), \operatorname{ccl} 17(p=0.0232)$ and $c c l 26(p=0.0296)$ and the receptors $c c r 3(p=0.0296), c c r 4(p=0.0416), c c r 5$ $(p=0.0110)$, ccr6 $(p=0.0273)$ and $c c r 8(p=0.0157)$ compared with the expression levels in asymptomatic animals (Fig. $3 \mathrm{~A}$ and $\mathrm{B}$ ). Furthermore, all these molecules were also downregulated compared with the expression levels in uninfected dogs. No differences in the $\mathrm{ccl} 3, \mathrm{ccl} 4, \mathrm{ccl} 5$, ccl20, ccl24, cxcl9 and cxcr3 mRNAs levels were founded in the hepatic tissue regardless of the clinical form displayed (Fig. 3A and B). The expression profiles of chemokines and their receptors in diseased animals showed a state
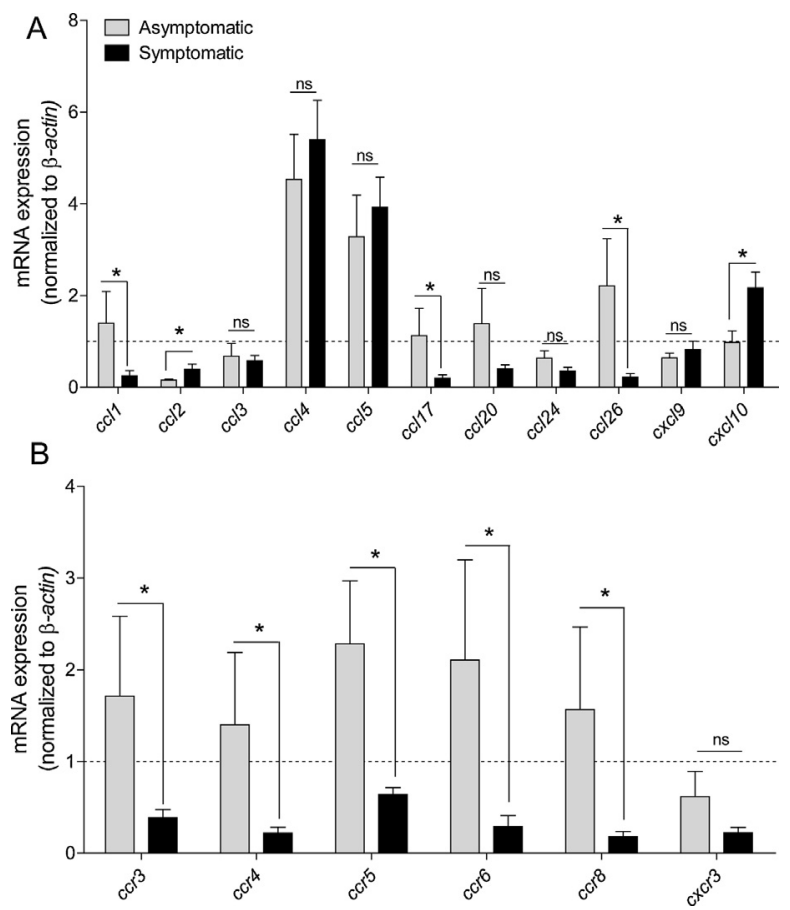

Fig. 3. Symptomatic dogs infected with Leishmania infantum exhibited reduced expression of chemokines and chemokine receptors in the liver. The mRNA expression levels of chemokines (A) and chemokine receptors (B) were determined by real-time PCR and normalized to the expression level of $\beta$-actin. The data are representative of ten $(n=10)$ symptomatic and eleven $(n=11)$ asymptomatic dogs per group and are expressed as the mean \pm SD. ${ }^{*} p<0.05$. ns: not significant. of immunosuppression due to the impairment of the migration of immune cells such as activated lymphocytes, monocytes, NK cells, B cells and dendritic cells, which can help with granuloma formation and parasite clearance.

We also analyzed the mRNA expression profiles of chemokines and chemokine receptors in the spleens of asymptomatic and symptomatic dogs. As already known, visceral Leishmania species induce an organ-specific immune response, making the response to this parasite even more complex (Mukherjee et al., 2003). In agreement with this immune response pattern, we detected distinct chemokine and chemokine receptor expression profiles in the spleens of animals with different clinical forms of the disease. The expression levels of $c c l 1, c c l 3, c c l 17, c c l 20$, ccl26, cxcl9, ccr3, ccr4, ccr6 and ccr8 were reduced in the spleens of symptomatic and asymptomatic dogs relative to the expression levels in uninfected animals (Fig. 4A and B). Interestingly, the mRNA levels of $c c l 2(p=0.0262)$, ccl5 $(p=0.0435)$ and $c x c l 10(p=0.0180)$ were increased in the spleens of symptomatic animals relative to the levels in the spleens of asymptomatic animals, and the mRNA level of cxcl10 was also upregulated compared with the level in uninfected dogs (Fig. 4A). Based on these results, we concluded that symptomatic dogs have lower overall expression levels of chemokines and chemokine receptors relative to asymptomatic and uninfected dogs. The impairment of cell migration favors parasite replication and spread and triggers the typical symptoms of CVL.

The results also indicated that there are negative correlations between the number of clinical signs and the ccr 5 and ccr6 mRNA expression levels in hepatic tissue (Fig. 5A and $\mathrm{B}$ ). In contrast, the correlation analysis showed that cxcl10 expression was positively associated with clinical status (Fig. 5 C); thus, the expression of this gene may be a compensatory mechanism for the low $c x c r 3$ expression.

\section{Discussion}

The involvement of the spleen and liver is a notable characteristic of $L$. infantum infection in vertebrate hosts. The parasitism of mononuclear phagocytic cells and liver hyperplasia and hypertrophy result in the constriction of capillaries, portal hypertension and ascites. Therefore, 


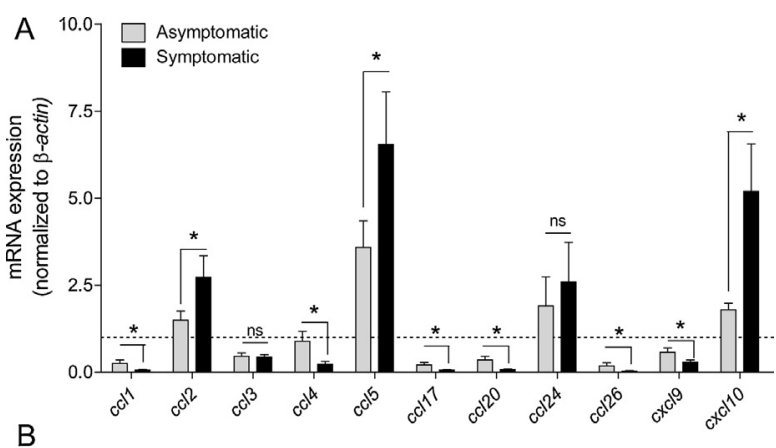

B

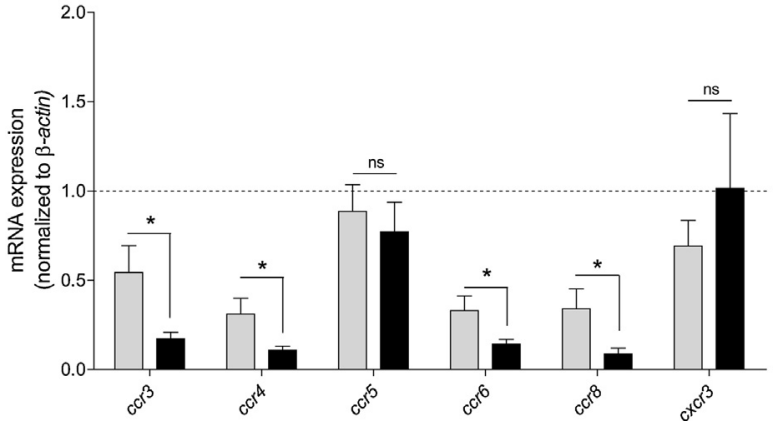

Fig. 4. Symptomatic and asymptomatic dogs infected with Leishmania infantum exhibited reduced expression of chemokines and chemokine receptors in the spleen. The mRNA expression levels of chemokines (A) and chemokine receptors (B) were determined by real-time PCR and normalized to the expression level of $\beta$-actin. The data are representative of ten $(n=10)$ symptomatic and eleven $(n=11)$ asymptomatic dogs per group and are expressed as the mean \pm SD. ${ }^{*} p<0.05$. ns: not significant.

studies that elucidate the immune responses against the parasite are clearly necessary. The analysis of the expression levels of chemokines and their receptors is crucial for assessing central regulation in homeostasis and the immunopathological mechanisms, including cell trafficking and inflammation, of VL in dogs and humans. Few studies have investigated the levels and/or the role of chemokines in CVL, and prior to this study, no studies had evaluated these molecules in the liver.

In this study, we showed that symptomatic naturally L. infantum-infected dogs displayed a clear impairment of the expression of chemokines and chemokine receptors in the liver compared with asymptomatic animals. The only exception was cxcl10, which was upregulated in livers and spleens of symptomatic animals and was also positively correlated with the clinical score. CXCL10 binds to CXCR3 to promote Th1 cell chemoattraction. However, we did not find statistically significant changes in cxcr3 expression; therefore, the high amount of this chemokine that is required for it to play its role is not achieved, and the parasite load increases. We hypothesized that increased cxcl10 expression occurs as a compensatory mechanism for the unchanged level of $c x c r 3$ expression. In agreement with these findings, it has been reported that dogs naturally or experimentally infected with $L$. infantum have upregulated cxcl10 mRNA expression in the spleen, leading to a substantial type- 1 immune response to CVL (Strauss-Ayali et al., 2007).

Like CXCL10, CCL5 is responsible for attracting inflammatory lymphocytes, particularly Th1-type cells, and macrophages. Previous studies have already shown that ccl5 mRNA expression is increased in naturally and experimentally L. infantum-infected dogs (Strauss-Ayali et al., 2007) and that there is a positive correlation between ccl5 expression and the parasite density in the skin (Menezes-Souza et al., 2012). We found the same results for the spleen, but ccr5 was downregulated in both groups of animals compared with controls, an effect that certainly impairs the chemokine activity. In hepatic tissue, despite the downregulation of ccr5, the expression level of which was negatively correlated with clinical status, no differences were observed in ccl5 expression between symptomatic and asymptomatic dogs, but both groups expressed significantly more $\mathrm{ccl} 5$ than uninfected animals.

Notably, the symptomatic animals expressed low levels of the ligands $c c l 1, c c l 17$, and $c c l 26$ and the receptors ccr8, ccr4, ccr3, and ccr6 in the liver. These genes were also severely downregulated in the spleen in both groups of animals compared with uninfected controls. CCL1, CCL17 and CCL26 are known to be important immunological markers of Th2 and/or regulatory T cells (Bromley et al., 2008). Both of these subsets are potent IL-10 producers and, therefore, are strongly related to immunosuppressive factors that often contribute to susceptibility and disease progression (Gautam et al., 2011; Ghalib et al., 1993; Nylen et al., 2007). Unexpectedly, the livers of symptomatic dogs expressed lower levels of these chemokines than animals with no symptoms. It is important to highlight the fact that the presence of these molecules in asymptomatic animals can be related to the regulation of exacerbated
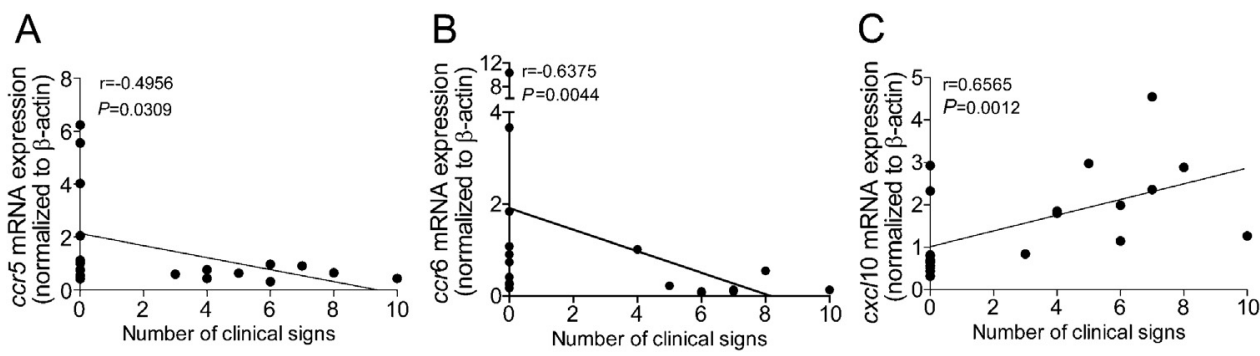

Fig. 5. Correlation between clinical status and the ccr5, ccr6 and cxcl10 mRNA expression levels. The results are expressed as scatter plots of individual values. The Spearman correlation indexes $(r)$ at $p<0.05$ are shown on the graphs. Connecting lines represent the positive and negative correlation indexes. 
inflammatory responses and can prevent liver damage. In fact, increased type 1 chemokine expression is correlated with cardiac pathology in dogs with canine Chagas disease due to myocardial inflammation (Guedes et al., 2010). In contrast, CCL20 and CCR6 are Th17-related molecules, i.e., inflammatory components. Indeed, IL-17, the primary Th17-related cytokine, has been demonstrated to have a protective role in human VL infections (Pitta et al., 2009). Hence, we can conclude that a delicate balance between inflammatory and anti-inflammatory stimuli is required to prevent symptoms.

In the spleen, the analysis showed that, compared with uninfected animals, both asymptomatic and symptomatic dogs had decreased levels of almost all chemokines and chemokine receptors that were analyzed. Despite this result, we observed that symptomatic animals had upregulated levels of $c c l 2, c c l 5$ and $c x c l 10$, and similar results were previously found in the spleens of experimentally L. infantum-infected dogs compared with uninfected dogs (Strauss-Ayali et al., 2007). In other studies, increased levels of $c c l 2$, ccl4, and $c c l 5$ were found in dogs with high levels of skin parasitism (Menezes-Souza et al., 2012). The increased expression of $\mathrm{ccl} 2, \mathrm{ccl} 5$ and $\mathrm{cxcl} 10$ in symptomatic animals is probably an attempt to compensate for both the absence of inflammatory cells in the spleen and the increased parasitism load. We did not observe differences in the ccl5 and ccl4 expression levels in the liver between the symptomatic and asymptomatic groups despite the fact that the infection inhibits $c c l 2$ and induces $c c l 4$ expression.

We also showed that $L$. infantum did not influence the expression of $\mathrm{ccl} 3$ or $\mathrm{cxcl9}$ (Th1-markers) in the livers of all infected animals as a mechanism of immune system evasion. It has already been reported that were no differences in the expression levels of $c c l 3$ between $L$. infantum-infected and uninfected dogs (Strauss-Ayali et al., 2007).

Altogether, our results demonstrate that symptomatic dogs exhibit an overall downregulation of the expression of chemokines and chemokine receptors, primarily in the liver, preventing the host from effectively defending against the parasite. Our findings also suggest that the chemokine and chemokine receptor expression profiles in the liver and spleen are extremely important for inducing the infiltration and accumulation of the correct cell type to effectively contain the infection and the disease. These findings represent an advance in the knowledge of the involvement of chemokines and their receptors in the response to CVL and may contribute to the understanding of the immunopathogenic mechanisms involved in the natural history of this disease.

\section{Acknowledgments}

This work was supported by CNPq -Conselho Nacional de Pesquisa e Desenvolvimento Científico e Tecnológico (Edital Universal: Processo 470772/2012-3), FAPERN Fundação de Amparo à Pesquisa do Estado do Rio Grande do Norte (Edital FAPERN 005/2011 PPP IV/Termo n ${ }^{0} 79$ ), and CAPES-Coordenação de Aperfeiçoamento de Pessoal de Ensino Superior (master and doctoral fellowships). LMCG received a visiting researcher fellowship from CNPq. We would like to thank the Center for Zoonosis Control in the Municipality of Mossoró, State of Rio Grande do Norte, Brazil, for the kind donation of the study animals.

\section{References}

Alves, C.F., de Amorim, I., Moura, E.P., Ribeiro, R.R., Alves, C.F., Michalick, M.S., Kalapothakis, E., Bruna-Romero, O., Tafuri, W.L., Teixeira, M.M., Melo, M.N., 2009. Expression of IFN-gamma, TNF-alpha, IL-10 and TGF-beta in lymph nodes associates with parasite load and clinical form of disease in dogs naturally infected with Leishmania (Leishmania) chagasi. Vet. Immunol. Immunopathol. 128, 349-358.

Ashford, R.W., 2000. The leishmaniases as emerging and reemerging zoonoses. Int. J. Parasitol. 30, 1269-1281.

Barbosa, M.A., exandre-Pires, G., Soares-Clemente, M., Marques, C., Rodrigues, O.R., De Brito, T.V., Da Fonseca, I., Alves, L.C., Santos-Gomes, G.M., 2011. Cytokine gene expression in the tissues of dogs infected by Leishmania infantum. J. Comp. Pathol. 145, 336-344.

Bromley, S.K., Mempel, T.R., Luster, A.D., 2008. Orchestrating the orchestrators: chemokines in control of $\mathrm{T}$ cell traffic. Nat. Immunol. 9, 970-980.

Chamizo, C., Moreno, J., Alvar, J., 2005. Semi-quantitative analysis of cytokine expression in asymptomatic canine leishmaniasis. Vet. Immunol. Immunopathol. 103, 67-75, 10-1-2005.

Desjeux, P., 2004. Leishmaniasis: current situation and new perspectives. Comp. Immunol. Microbiol. Infect. Dis. 27, 305-318.

Dietze, R., Barros, G.B., Teixeira, L., Harris, J., Michelson, K., Falqueto, A., Corey, R., 1997. Effect of eliminating seropositive canines on the transmission of visceral leishmaniasis in Brazil. Clin. Infect. Dis. 25, 1240-1242.

Gautam, S., Kumar, R., Maurya, R., Nylen, S., Ansari, N., Rai, M., Sundar, S., Sacks, D., 2011. IL-10 neutralization promotes parasite clearance in splenic aspirate cells from patients with visceral leishmaniasis. J. Infect. Dis. 204, 1134-1137, 1-10-2011.

Ghalib, H.W., Piuvezam, M.R., Skeiky, Y.A., Siddig, M., Hashim, F.A., elHassan, A.M., Russo, D.M., Reed, S.G., 1993. Interleukin 10 production correlates with pathology in human Leishmania donovani infections. J. Clin. Invest. 92, 324-329.

Giunchetti, R.C., Mayrink, W., Genaro, O., Carneiro, C.M., Correa-Oliveira, R., Martins-Filho, O.A., Marques, M.J., Tafuri, W.L., Reis, A.B., 2006. Relationship between canine visceral leishmaniosis and the Leishmania (Leishmania) chagasi burden in dermal inflammatory foci. J. Comp. Pathol. 135, 100-107.

Guedes, P.M., Veloso, V.M., Talvani, A., Diniz, L.F., Caldas, I.S., Do-ValleMatta, M.A., Santiago-Silva, J., Chiari, E., Galvao, L.M., Silva, J.S., Bahia, M.T., 2010. Increased type 1 chemokine expression in experimental Chagas disease correlates with cardiac pathology in beagle dogs. Vet. Immunol. Immunopathol. 138, 106-113, 15-11-2010.

Mauricio, I.L., Stothard, J.R., Miles, M.A., 2000. The strange case of Leishmania chagasi. Parasitol. Today 16, 188-189.

Menezes-Souza, D., Correa-Oliveira, R., Guerra-Sa, R., Giunchetti, R.C., Teixeira-Carvalho, A., Martins-Filho, O.A., Oliveira, G.C., Reis, A.B., 2011. Cytokine and transcription factor profiles in the skin of dogs naturally infected by Leishmania (Leishmania) chagasi presenting distinct cutaneous parasite density and clinical status. Vet. Parasitol. 177, 39-49.

Menezes-Souza, D., Guerra-Sa, R., Carneiro, C.M., Vitoriano-Souza, J., Giunchetti, R.C., Teixeira-Carvalho, A., Silveira-Lemos, D., Oliveira, G.C., Correa-Oliveira, R., Reis, A.B., 2012. Higher expression of CCL2, CCL4, CCL5, CCL21, and CXCL8 chemokines in the skin associated with parasite density in canine visceral leishmaniasis. PLoS. Negl. Trop. Dis. 6, e1566-e1570.

Moreno, J., Alvar, J., 2002. Canine leishmaniasis: epidemiological risk and the experimental model. Trends Parasitol. 18, 399-405.

Mott, K.E., Desjeux, P., Moncayo, A., Ranque, P., de Raadt, P., 1990. Parasitic diseases and urban development. Bull. World Health Organ. 68, 691-698.

Mukherjee, P., Ghosh, A.K., Ghose, A.C., 2003. Infection pattern and immune response in the spleen and liver of BALB/c mice intracardially infected with Leishmania donovani amastigotes. Immunol. Lett. 86, 131-138, 3-4-2003.

Nascimento, E.L., Martins, D.R., Monteiro, G.R., Barbosa, J.D., Ximenes, M.F., Maciel, B.L., Duarte, I., Jeronimo, S.M., 2008. Forum: geographic spread and urbanization of visceral leishmaniasis in Brazil, Postscript: new challenges in the epidemiology of Leishmania chagasi infection. Cad. Saude Publica 24, 2964-2967. 
Nylen, S., Maurya, R., Eidsmo, L., Manandhar, K.D., Sundar, S., Sacks, D., 2007. Splenic accumulation of IL-10 mRNA in T cells distinct from $\mathrm{CD} 4+\mathrm{CD} 25+($ Foxp3 $)$ regulatory T cells in human visceral leishmaniasis. J. Exp. Med. 204, 805-817, 16-4-2007.

Pitta, M.G., Romano, A., Cabantous, S., Henri, S., Hammad, A., Kouriba, B., Argiro, L., el Kheir, M., Bucheton, B., Mary, C., El-Safi, S.H., Dessein, A., 2009. IL-17 and IL-22 are associated with protection against human kala azar caused by Leishmania donovani. J. Clin. Invest. 119, 2379-2387.
Reiner, S.L., Locksley, R.M., 1995. The regulation of immunity to Leishmania major. Annu. Rev. Immunol. 13, 151-177.

Strauss-Ayali, D., Baneth, G., Jaffe, C.L., 2007. Splenic immune responses during canine visceral leishmaniasis. Vet. Res. 38, 547-564.

Travi, B.L., Tabares, C.J., Cadena, H., Ferro, C., Osorio, Y., 2001. Canine visceral leishmaniasis in Colombia: relationship between clinical and parasitologic status and infectivity for sand flies. Am. J. Trop. Med. Hyg. 64, 119-124. 